\title{
Measurement of the electron neutrino component of the T2K beam in the ND280 Tracker
}

\section{Davide Sgalaberna* for the T2K Collaboration}

ETH Zurich, Switzerland

E-mail: davide.sgalaberna@cern.ch

\begin{abstract}
The main physics goal of the T2K experiment is the measurement of the PMNS matrix through the observation of $v_{e}$ appearance in the $v_{\mu}$ neutrino beam. The main background to this measurement is the intrinsic $v_{e}$ beam component that has to be measured before the oscillation at the T2K Near Detector (ND280). We select neutrino interactions in the Fine Grained Detector (FGD) and distinguish electrons from muons by combining the tracking and PID capabilities of 3 Time Projection Chambers (TPC) and an Electromagnetic Calorimeter (ECAL). Thanks to the combination of these detectors, we are able to reject more than $99 \%$ of the muons produced by the dominant $v_{\mu}$ interactions. We will show details of the detector performances and selection analysis. Finally a comparison of the selected data with the MC expectation will be presented. The result of this analysis provides confidence in the understanding of the intrinsic $v_{e}$ beam component of the T2K beam simulation and of the most recent $\mathrm{T} 2 \mathrm{~K} v_{e}$ appearance results.
\end{abstract}

The European Physical Society Conference on High Energy Physics

18-24 July, 2013

Stockholm, Sweden

${ }^{*}$ Speaker. 

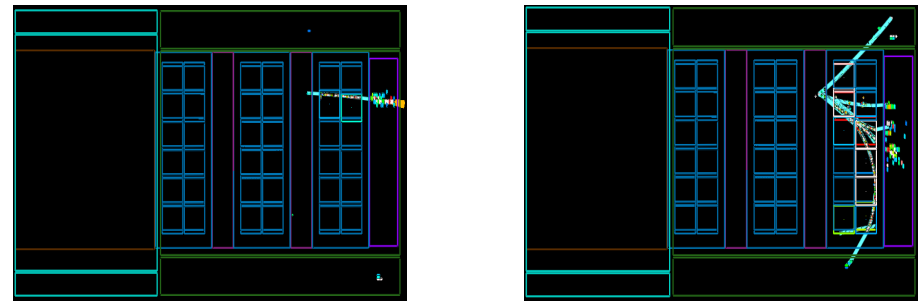

Figure 1: CCQE (left) and CCnQE (right) candidates selected at ND280.

\section{The T2K experiment}

The T2K (Tokai-to-Kamioka) experiment is a long baseline neutrino oscillation experiment that aims to measure the PMNS matrix through the observation of $v_{\mu} \rightarrow v_{e}$ appearance.

The neutrino beam is generated by a high intensity $31 \mathrm{GeV} / \mathrm{c}$ proton beam interacting on a carbon target and is detected by a near detector (ND280), $280 \mathrm{~m}$ from the target, and a far detector located $295 \mathrm{~km}$ away (Super Kamiokande). The neutrino beam is composed of about $99 \%$ of $v_{\mu}$ and the remaining $1 \%$ of $v_{e}$ [1]. This beam $v_{e}$ component represents the main, irreducible background to the $v_{e}$ appearance signal at Super-Kamiokande and it has to be measured at ND280 before the oscillations. For this analysis all the T2K data collected until April 2013 are used and it correspond to a statistics of $5.9 \times 10^{20}$ p.o.t.

\section{Measurement of the $v_{e}$ beam contamination at ND280}

For this analysis the ND280 Tracker is used: neutrinos interact in the fully-active scintillator Fine-Grained Detectors (FGDs). The charged particles produced in the neutrinos interactions enter then the Time Projection Chambers (TPC) where the charge and the momentum of the particle is reconstructed. Given the small beam $v_{e}$ component the PID capabilities of ND280 have to be used to distinguish electrons from muons: the PID is based on the measurement of the energy loss in TPCs (deposited energy resolution of $8 \%$ ) combined with the information from the surrounding Electromagnetic Calorimeter (ECAL) where the electrons produce a shower. In this way a muon rejection factor of $99.8 \%$ is reached and a very clean sample of electrons is selected. Looking at the topology of the events, the selection can be further improved, separating Charged Current Quasi Elastic (CCQE) interactions $\left(v_{e}+n \rightarrow e^{-}+p\right)$ from Charged Current non Quasi Elastic (CCnQE) $\left(v_{e}+n \rightarrow e^{-}+p+X\right)$ (see figure 1). Those two samples are separated by looking at the number of reconstructed tracks in TPC, FGD and ECAL and to the detection of Michel electrons.

In figure 2 the selected $\mathrm{CCQE}$ and $\mathrm{CCnQE}$ samples are shown. A pure sample of electrons is selected with a purity of $67 \%$. The main background comes from photon conversion that dominates the low momentum region below $500 \mathrm{MeV}$. For this reason it is fundamental to constrain this background selecting a photon control sample directly from the data. A pure sample is obtained selecting $e^{+} e^{-}$pairs entering the TPC by reconstructing their invariant mass (see figure 3 ).

The $v_{e}$ beam contamination at ND280 is measured performing a likelihood fit on the three 

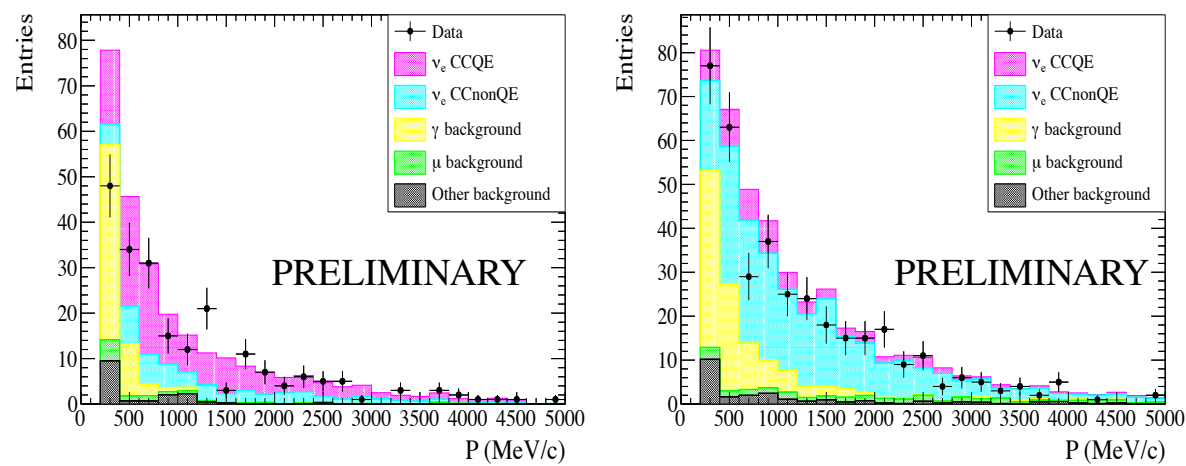

Figure 2: Reconstructed electron momentum for the events selected after applying CCQE (left) and CCnQE (right) selections.

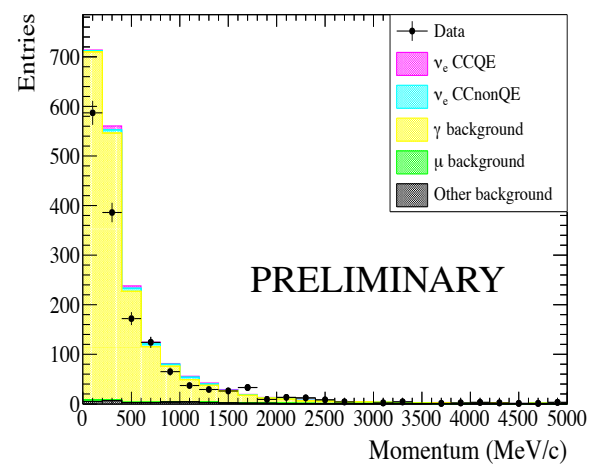

Figure 3: Reconstructed electron momentum after applying the photon selection.

samples and the scaling for the $v_{e}$ beam component is:

$$
f^{v_{e}}=1.055 \pm 0.58 \text { (stat.) } \pm 0.074 \text { (syst.) }
$$

With the systematics coming from detector (5.0\%) and flux and $\mathrm{x}$-sec systematics (5.8\%). This is a very important confirmation that the $v_{e}$ contamination in the T2K beam is well understood. When more statistics will be available, the selected sample will also be used to further constrain the flux and cross section parameters for the T2K oscillation analyses and it will also be used to perform sterile searches and measurements of electron neutrino cross-sections.

\section{References}

[1] K. Abe et al., Nucl. Instr. and Meth. A 659 (1) (2011) 106-135, arXiv:1106.1238v2 